\title{
The Microwave-assisted Lipase-catalyzed Synthesis of Sucrose Laurate
}

\author{
Quanhui $\mathrm{Li}^{1, \mathrm{a}}$, Jiaying Xin ${ }^{1, \mathrm{~b}^{*}}$, Tingting $\mathrm{Yao}^{1, \mathrm{c}}$, \\ Zhengyang Wang ${ }^{1, d}$ and Luoyun Zheng ${ }^{1, e}$ \\ ${ }^{1}$ Key Laboratory for Food Science and Engineering, Harbin University of Commerce,China \\ a1023281117@qq.com, binjiayingvip@163.com, 657407999@qq.com, \\ d306224963@qq.com, ${ }^{e} 506637547 @ q q . c o m$
}

Keywords: Sugar ester; Lipase; Enzymatic non-aqueous system.

\begin{abstract}
In this study, the lauroyl sucrose ester was synthesized by microwave-assisted lipase-catalyzed reaction under non-aqueous system. The influence of the microwave power, the amount of molecular sieve added and the reaction time on the conversion of sugar esters were explored. The results showed that compared with conventional heating, microwave-assisted lipase-catalyzed method was short time and can reduce energy consumption. And the thin layer chromatography showed that the lauroyl sucrose ester synthesized by microwave-assisted lipase-catalyzed reaction containing only a single product spot. The optimal conditions was built when the reaction was carried out under $200 \mathrm{~W}$ of the microwave reaction power and $15 \mathrm{~g} / \mathrm{L}$ of molecular sieve for $25 \mathrm{~min}$. The maximum conversion of lauroyl sucrose ester was $63.8 \%$ under the optimal condition. After three times batch reaction, the residual activity of lipase was $52.4 \%$.
\end{abstract}

\section{Introduction}

Microwave heating is a valuable tool for synthetic chemists. It is capable of improving product yields and enhancing the rate of reactions as well as being a safe and convenient method for heating reaction mixtures to elevated temperatures. Domestic microwave ovens are increasingly being replaced by scientific microwave apparatus for use in synthesis [1]. Microwave heating occurs on a molecular level as opposed to relying on convection currents and thermal conductivity when using conventional heating methods. It is possible to perform reactions at modest reaction temperatures and obtain great improvements in rate and yield [2]. The advantages of microwave-assisted organic synthesis (MAOS) include reduced chemical reaction time, reduced side reactions, increased yields and improved reproducibility [3]. Microwave irradiation is an approach for process intensification in a variety of reactions [4]. Microwave irradiated synthesis in organic solvents using both chemical and biocatalysis means has received significance in green process development. Under microwave irradiation, high yields and reaction selectivity can be achieved at reduced reaction time. Microwave irradiation results in an instantaneous localized superheating which is achieved due to dipole rotation or ionic conduction. Microwave irradiation has been applied to the synthesis of several natural products as well as peptides, peptoids and carbohydrates [5-8].

Sucrose esters are the largest and most widely used in industrial production. Sucrose esters in industrial production are usually mixtures with different degrees of esterification, but the degree of esterification is usually less than 4. Sugar fatty acid esters can be produced by non-enzymatic chemical routes. The chemical routes require more energy than the enzymatic route and have a low selectivity. In addition, the combination of high temperature and alkaline catalyst used in chemical processes, causes discoloration of the product and the formation of toxic byproducts. Some of the byproducts are allergenic and possibly carcinogenic [9]. Synthesis can be performed under mild reaction conditions, which are compared to the chemical synthesis to minimize side reactions and the browning of the products. As the catalysts in organic synthesis, lipases not only catalyze hydrolysis but also esterification, transesterification and so on. Each lipase demonstrates its distinct substrate specificity, regioselectivity and steroselectivity. Enzyme-catalyzed reactions are superior to 
conventional chemical methods because of mild reaction conditions, high catalytic efficiency and the inherent selectivity of natural catalysts. Further, the use of immobilized enzyme can simplify the downstream processing [10]. In this experiment, the combination of microwave irradiation and lipase catalyzed were established. The effects of microwave power, reaction time and the adding amount of molecular sieve on the rate of sugar ester reaction were studied. And the reaction process of the non-aqueous phase lipase-catalyzed synthesis of sugar esters was observed. The reaction condition of microwave-assisted lipase-catalyzed synthesis of sucrose laurate was optimized.

\section{Materials and Methods}

Materials. Novozym435 lipase was purchased from Denmark Novozymes A/S Co. Lauric acid and sucrose was purchased from Suzhou open chemical Co., Ltd. All other chemicals were obtained commercially and were analytical grade. tert-butanol, methylbenzene, methanol, ethyl acetate, phenolphthalein, potassium hydroxide, ethy alcohol was purchased from Tianjin Tianli Chemical Reagent Co., Ltd.

Synthesis of Sucrose Esters by Microwave-assisted Lipase-catalyzed Reaction. $10 \mathrm{~mL}$ of 0.6 $\mathrm{mol} / \mathrm{L}$ lauric acid tert-butanol solution, $0.08 \mathrm{~mol} / \mathrm{L}$ sucrose, $30 \mathrm{~g} / \mathrm{L}$ Novozym 435 lipase, $0 \sim 60 \mathrm{~g} / \mathrm{L}$ of molecular sieve were added together and reaction was carried out at $30 \sim 50{ }^{\circ} \mathrm{C}, 160 \sim 260 \mathrm{~W}$ for 10 60min in a microwave chemical reactor.

Determination of Lauric acid Sucrose Ester Conversion. $0.5 \mathrm{~mL}$ of reaction solution was added 1 drop of $0.1 \%$ phenolphthalein indicator after completion of the reaction, with $0.025 \mathrm{~mol} / \mathrm{L}$ potassium hydroxide solution to titrate the remaining lauric of reaction system. Sugar esters conversion was calculated according to the lauric acid consumption of reaction (In monoester count). Parallel determination three times and averaged [10]. Formula is as follows: conversion of sugar ester $(\%)=($ Consumption of fatty acids $) /($ The amount of sugar was added $) \times 100 \%$

Infrared Spectroscopic Analysis of Sugar Esters. Infrared spectroscopic analysis of sugar esters was through the method of potassium bromide. Sugar esters after vacuum dried for $24 \mathrm{~h}$ was milled together with dry potassium bromide powder, into the tablet device for $5 \mathrm{~min}$. Then scans the sample by infrared spectrometer to observe the absorption wavelength range of each band. The characteristic frequency of the functional group pointed out that the presence of functional groups in the molecule, in order to determine the samples chemically bonded form. Scan test was carried out with resolution $4 \mathrm{~cm}-1$, the number of scans was 8 times and the wavelength scan range of $450 \sim 4000 \mathrm{~cm}-1$.

\section{Results}

Effect of Microwave Power on the Microwave-assisted Lipase-catalyzed Reaction. Microwave-assisted lipase-catalyzed reaction was effected through thermal effects and non-thermal effects of microwave. Since the new type of microwave reactor can be controlled to achieve the constant temperature parameters, so it can achieve constant temperature under different power. Suitable microwave intensity can enhance the movement of the substrate molecule, increase opportunities for substrate molecules collide and to some extent, "activation" of the lipase, accelerate the esterification reaction. However, if the microwave power is too large, it will accelerate the kinetic energy of the enzyme molecule groups, so that over hydrogen-bond bonding energy, lead to hydrogen-bond rupture, the enzyme molecule conformation destruction, the activity decreased. And at constant temperature, the power is so large that microwave chemical reactor working arrest sudden breaks, does not help to extend the life of a microwave reactor.

In this experiment, $0.6 \mathrm{~mol} / \mathrm{L}$ of lauric acid, $0.08 \mathrm{~mol} / \mathrm{L}$ of sugar added, $30 \mathrm{~g} / \mathrm{L}$ of lipase added, $60 \mathrm{~g} / \mathrm{L}$ of molecular sieve were added together, the reaction solvent was t-butanol, and the temperature was at $50^{\circ} \mathrm{C}$. The effect of microwave power on the conversion of lauric acid sucrose ester was investigate. The result was shown in Fig. 1 


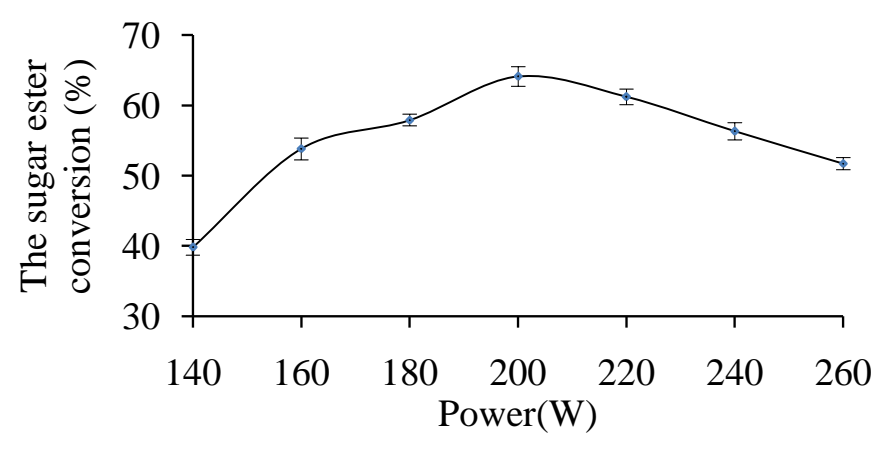

Figure 1. The effect of microwave power on conversion of sugar ester

In this study, by means of a direct reaction conventional heating system, the effect of microwave power on conversion of lauric acid sucrose ester was investigated at reaction for $20 \mathrm{~min}$. As can be seen from the Fig. 1, microwave power has a great influence on the conversion of sucrose ester. When the microwave power was $140 \mathrm{~W}$, the conversion of sucrose ester was low. The conversion of sucrose esters is increasing gradually with microwave power increased. When the microwave power increased to $200 \mathrm{~W}$, the conversion of sucrose ester was the maximum, which was $64.1 \%$. With the further increase of microwave power, the conversion of sugar esters began to decrease. It may be that the microwave power is too large, so that the loss of some of the lipase activity.

Effect of Molecular Sieve Added on the Conversion Rate of Sucrose Laurate. Water is a product of the esterification reaction. As the reaction proceeds and the accumulation of water, the reaction will be the reverse, so that sucrose ester will be hydrolyzed. The same as the conventional heating reaction, microwave reaction needs to control the amount of water in the reaction system. Different from the conventional heating, water is a polar molecule, and the dielectric constant is very large, and the water molecules are obviously affected by the microwave electromagnetic field. Under microwave irradiation, water molecules may be separated from the reaction system, which is favorable for the synthesis of sugar esters catalyzed by lipase in the non-aqueous phase. So the effect of 4A zeolite addition on the conversion of lauric acid sugar esters were studied to verify the effect of microwave method on water content in the system. Lauric acid concentration was $0.6 \mathrm{~mol} / \mathrm{L}$, the amount of sugar added was $0.08 \mathrm{~mol} / \mathrm{L}$, the amount of lipase was $30 \mathrm{~g} / \mathrm{L}$, reaction solvent was t-butanol, microwave power was $200 \mathrm{~W}$, reaction temperature was $50{ }^{\circ} \mathrm{C}$, reaction time was $25 \mathrm{~min}$.

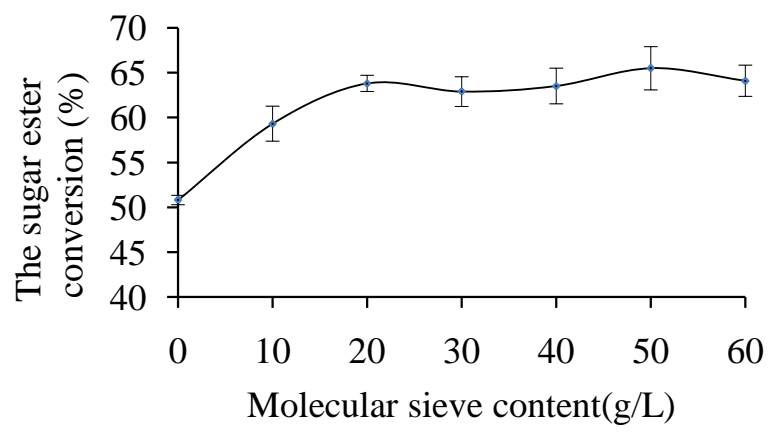

Figure 2. Effect of zeolite addition on the conversion of sucrose laurate

As shown in Fig. 2. When the molecular sieve was not added, the conversion of sucrose ester could reach $50.8 \%$. With the increase of the amount of molecular sieve, the conversion of sucrose esters of lauric gradually increased, when the amount of molecular sieve reaches $20 \mathrm{~g} / \mathrm{L}$, with the further increasing amount of molecular sieve added the conversion of sugar esters no significant change. Due to the microwave-assisted lipase-catalyzed reaction using magnetic stirring method, too much of the 
molecular sieve will cause the reaction liquid splash. Therefore, $20 \mathrm{~g} / \mathrm{L}$ was used as the amount of $4 \mathrm{~A}$ molecular sieve. It can be seen that the microwave radiation is indeed contribute water molecules from the reaction system, as compared with the conventional heating method, the amount of molecular sieve can be saved.

\section{Effect of Reaction Time on the Conversion of Sucrose Laurate.}

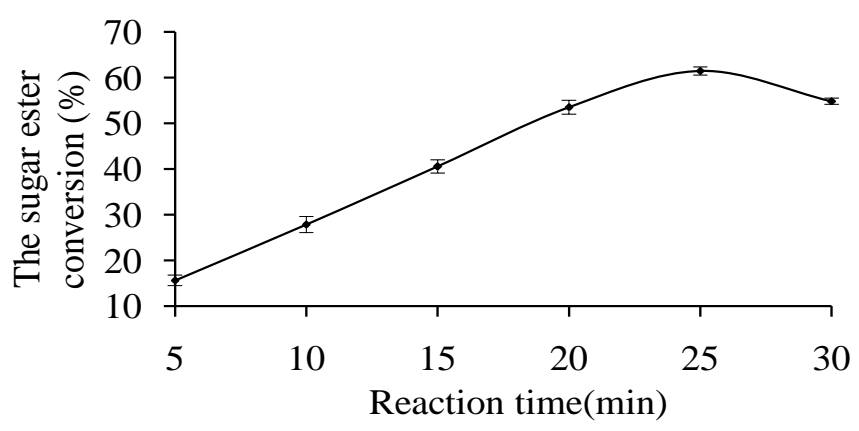

Figure 3. Effect of reaction time on the conversion of sucrose laurate

The reaction time is an important parameter affecting the reaction equilibrium. For microwave method, the length of time is related to the amount of radiation of microwave. The proper amount of microwave radiation not only helps to increase the molecular motion of the substrate, but also can stimulate the activity of the enzyme to a certain extent. While excessive microwave radiation can cause enzyme activity weakened. By this experiment, lauric acid concentration was $0.6 \mathrm{~mol} / \mathrm{L}$, the amount of sucrose added was $0.08 \mathrm{~mol} / \mathrm{L}$, the added amount of lipase was $30 \mathrm{~g} / \mathrm{L}$, amount of molecular sieve was $20 \mathrm{~g} / \mathrm{L}$, the reaction solvent is t-butanol, the microwave power was $200 \mathrm{~W}$, the reaction temperature was $50{ }^{\circ} \mathrm{C}$. Under the conditions, the effect of reaction time on the conversion of sucrose ester was studied. It can be seen from the Fig. 3, as time increases, the conversion of lauric acid sucrose esters increases within $5 \sim 25 \mathrm{~min}$ range, the reaction rate of each time period approximately equal. When the time was over $25 \mathrm{~min}$, the conversion of sucrose esters decreased. It may be too long because microwave radiation, changing the enzyme protein secondary conformation, caused by peptide bond cleavage, lipase lost some vigor. So the reaction time should be selected in about $25 \mathrm{~min}$ appropriate.

Infrared Spectrum Analysis of Lauric Acid Sucrose Ester.

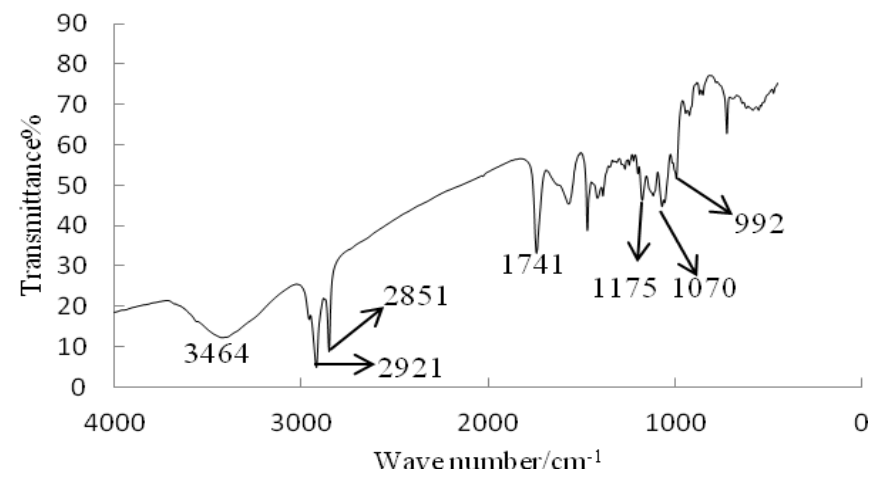

Figure 4. Infrared spectra of lauric acid sucrose ester

As shown in Fig. 4, an obvious absorption peak was appeared in $1741 \mathrm{~cm}-1$, which is the characteristic absorption peak of ester bond. Synthesis of sucrose esters by microwave method was also proved by the results of the thin layer chromatography. Other absorption peaks: associating 
hydroxyl stretching vibration absorption peak at $3464 \mathrm{~cm}-1, \mathrm{C}-\mathrm{H}$ stretching vibration absorption peak at $2921 \mathrm{~cm}-1,2851 \mathrm{~cm}-1$, sucrose cyclic structure C-O stretching vibration absorption peak at 1175 cm-1, $1070 \mathrm{~cm}-1$, glycoside bond characteristic absorption peaks at $992 \mathrm{~cm}-1$. These are the characteristic absorption peaks of sucrose esters.

Microwave - Residual Activity of Lipase-coupled

Table 1 Lipase Residual Vitality

\begin{tabular}{ll}
\hline Experiment times & Residual enzyme activity \\
\hline 1 & $100 \%$ \\
2 & $70.3 \%$ \\
3 & 54.2 \\
\hline
\end{tabular}

As can be seen from the Table 1, compared with the conventional heating method, the same number of lipase use, the residual enzyme activity of microwave method is low, which shows that the effect of microwave radiation on the activity of lipase.

\section{Summary}

In our study, we used microwave-assisted lipase-catalyzed method instead of conventional heating to synthesis lauric acid sucrose ester. The effects of reaction power, molecular sieve addition and reaction time on the conversion of sugar esters were investigated. By infrared spectroscopy product qualitative analysis proved the product was monolaurate. Compared with conventional heating, microwave catalytic method has a short time, can reduce energy consumption, the product does not have two esters. However, the method of microwave catalysis has a great influence on the stability of lipase activity.

\section{References}

[1] J. Gising, LR Odell, M Larhed, Microwave-assisted synthesis ofsmall molecules targeting the infectious diseases tuberculosis, HIV/AIDS, malaria and hepatitis C, Org Biomol Chem. 10( 2012) 2713-29.

[2] Y. Shen, W. Guo, L. Qi, J. Qiao, F. Wang, L. Mao, Immobilization of trypsin via reactive polymer grafting from magnetic nanoparticles for microwave-assisted digestion, J. Mater Chem B. 1(2013)2260-7.

[3] Appukkuttan P, Mehta VP, Van der Eycken EV, Microwave-assisted cycloaddition reactions, Chem Soc Rev. 39(2010)1467-77.

[4] GD. Yadav, SD. Shinde, Process intensification of immobilized lipase catalysis by microwave irradiation in the synthesis of4-chloro-2-methylphenoxyacetic acid (MCPA) esters. Biochem Eng J. 90(2014)96-102.

[5] Horchani H, Chaâbouni M, Gargouri Y, Sayari A. Solvent-free lipasecatalyzed synthesis of long-chain starch esters using microwave heating: optimization by response surface methodology, Carbohydr Polym. 79( 2010)466-74.

[6] Yadav GD, Thorat PA. Microwave assisted lipase catalyzed synthesis ofisoamyl myristate in solvent-free system, J. Mol Catal B: Enzym. 83(2012)16-22.

[7] Kappe OC, Pieber B, Dallinger D. Microwave effects in organic synthesis: myth or reality? Angew Chem Int Ed. 52(2013) 1088-94.

[8] Young DD, Nichols J, Kelly RM, Deiters A. Microwave activation of enzymatic catalysis, J Am Chem Soc. 130(2008)10048-9. 
[9] Gumel A. M, Annuar M S M, Heidelberg T, et al. Lipase mediated synthesis of sugar fatty acid esters, Process Biochemistry. 46(2011)2079-2090.

[10] Yu J, Zhang J, Zhao A et al. Study of glucose ester synthesis by immobilized lipase from Candida sp, Catalysis Communications. 9(2008)1369-1374. 